\title{
BASIC LIFE SUPPORT
}

\author{
Revised recommendations of the Resuscitation Council (UK)
}

\author{
A K Marsden
}

In 1982 the Resuscitation Council (UK) published guidelines for basic life support. The reconvened basic life support working group now includes senior medical advisers to the voluntary aid societies, and it has consulted representatives of the nursing profession and the National Health Service Training Authority (which is responsible for the training syllabus of the ambulance service.) Having reviewed published research, the working group believes that certain key changes in the guidelines are necessary, particularly in respect of expired air respiration.

The Resuscitation Council has also produced revised guidelines for advanced life support, described in the accompanying paper ( $p 446$ ).
Most cardiac arrests in the community are the result of ischaemic heart disease. Forty per cent of sudden deaths from ischaemic heart disease occur within one hour after the onset of symptoms, and the proportion is higher-around $60 \%$ - among middle aged and younger men. Over $90 \%$ of the deaths which occur outside hospital are due to ventricular fibrillation, a potentially reversible condition.

Survival from cardiac arrest is optimal when:

- the event is witnessed

- a bystander starts resuscitation

- the rhythm is ventricular fibrillation

- defibrillation is carried out at an early stage. ${ }^{1}$ The emphasis should be on early defibrillation and, irrespective of other considerations, defibrillators should be made available more widely for the use of suitably trained staff.

\section{Levels of knowledge}

"Basic life support" refers to maintaining an airway and supporting breathing and circulation without any equipment. Basic life support skills should be learnt by everyone, including schoolchildren. The techniques of basic life support are no different when practised by health care professionals, but they should also be expected to practise more complex skills, including the use of airway and ventilatory adjuncts, and two rescuer cardiopulmonary resuscitation. The extra, advanced skills used by the professional are included here for completeness but will require differentiation in resuscitation training manuals.

\section{Assessment and airway control}

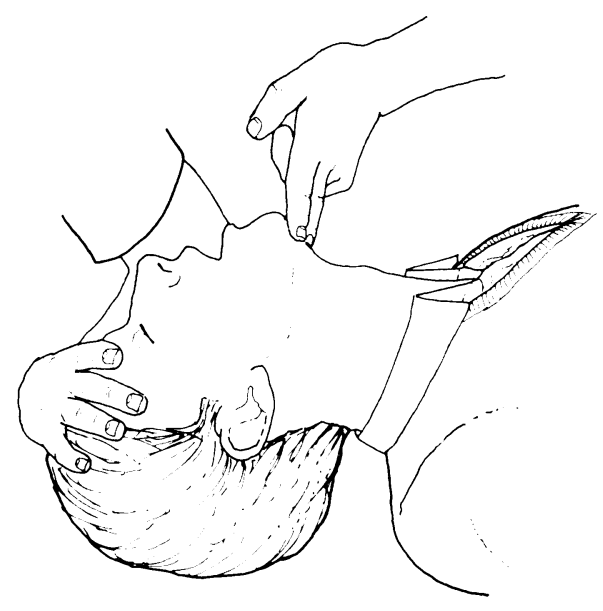

Members of the public should be aware of the importance of calling an ambulance immediately in cases of sudden collapse. They should be encouraged to summon professional help before starting or helping in prolonged cardiopulmonary resuscitation.

The rescuer should demonstrate unresponsiveness by shaking the casualty carefully and asking, "What's happening?" or "Are you all right?" If the patient is unresponsive the rescuer should call for help. The ABC ( $A$ irway, $B$ reathing, $C$ irculation) order of assessment and resuscitation is recommended for the lay rescuer. Occasionally a single rescuer's initial attempts may be rewarded by the return of respiration or a spontaneous heartbeat.

The rescuer should "open the airway" by the combined maneouvre of head tilt and chin lift. In most cases this alone will lift the tongue from the back of the throat. ${ }^{2}$ Any obvious obstruction should be removed from the mouth. This includes broken or displaced dentures, but well fitting dentures help to maintain a mouth seal during ventilation and should not be removed. 


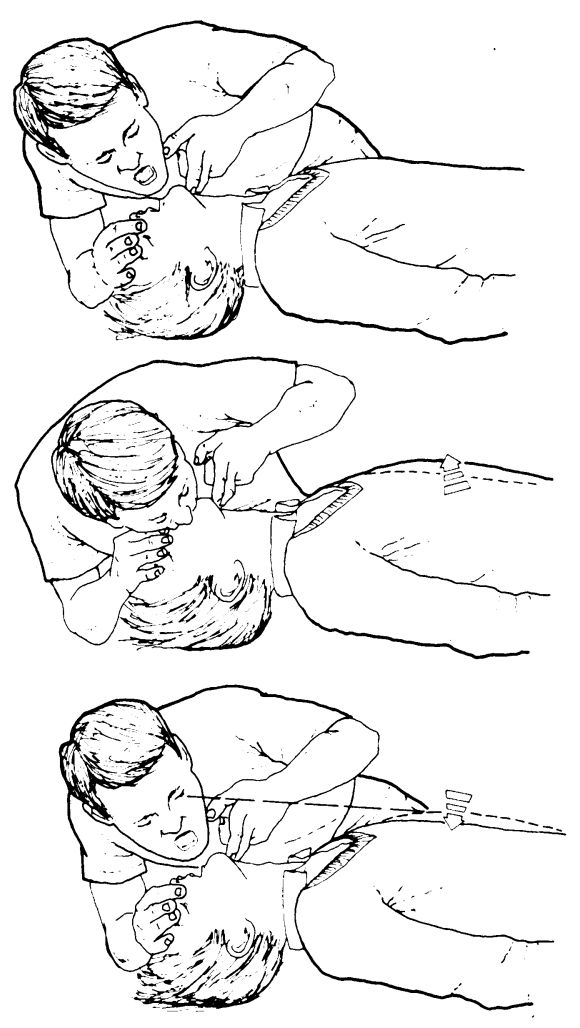

Once the airway has been opened the rescuer should check for breathing by looking for chest movements, listening for breath sounds, and feeling for the exhaled air on the back of the hand. "Breathing" in basic life support is provided by the expired air method.

There is now evidence that giving breaths rapidly in expired air respiration causes air to enter the stomach. This increases the likelihood of regurgitation and aspiration of stomach contents. ${ }^{3}$ We agree with the recommendations of the American Heart Association that at the beginning of resuscitation of the apnoeic patient there should be two slow expired breaths of air, each sufficient to cause the chest to rise, ${ }^{4}$ rather than the four rapid breaths previously recommended. The chest should be seen to fall between ventilations. Each of the initial breaths should last from one to one and a half seconds. Succeeding ventilations should, likewise, last long enough to allow the chest to rise.

Mouth to mouth respiration is usually preferred, but for resuscitation in water the mouth to nose method may be more practicable. Exhalation with the mouth to nose method should, however, be through the mouth as the nasal tissues often obstruct passive exhalation.

Members of the public should not be afraid of performing resuscitation. Mouth to mouth resuscitation carries no known risk of the transmission of the HIV or hepatitis B virus. There is no evidence that HIV has been transmitted by saliva. It is important to remember that up to $70 \%$ of cardiac arrests occur in the home and involve people who will be known to the rescuer and who might be family members, neighbours, or close friends. People who have performed resuscitation who, despite such reassurances, are still concerned at the possibility of disease transmission should seek further professional advice.

\section{Circulation}

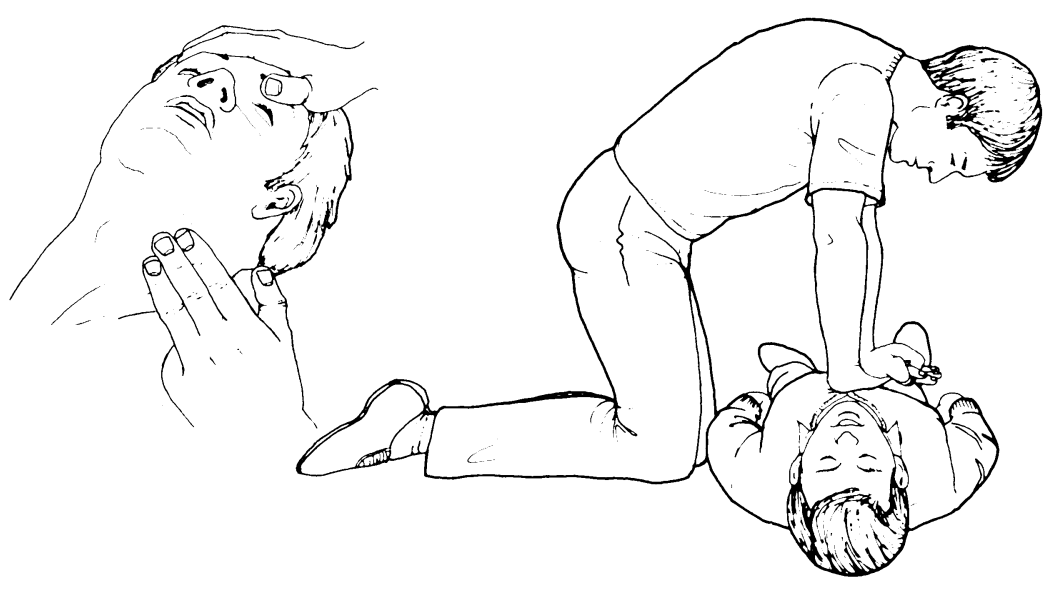

Basic life support training materials should emphasise the importance of checking the pulse properly. The carotid pulse should be palpated for at least five seconds to ensure that the circulation has stopped.

For chest compressions to be effective and delivered without undue fatigue a rate of $60-80$ per minute is recommended. This rate can be achieved by counting aloud "one and," "two and," etc. For single rescuer cardiopulmonary resuscitation the compression to ventilation ratic remains 15:2.

\section{Recovery position}
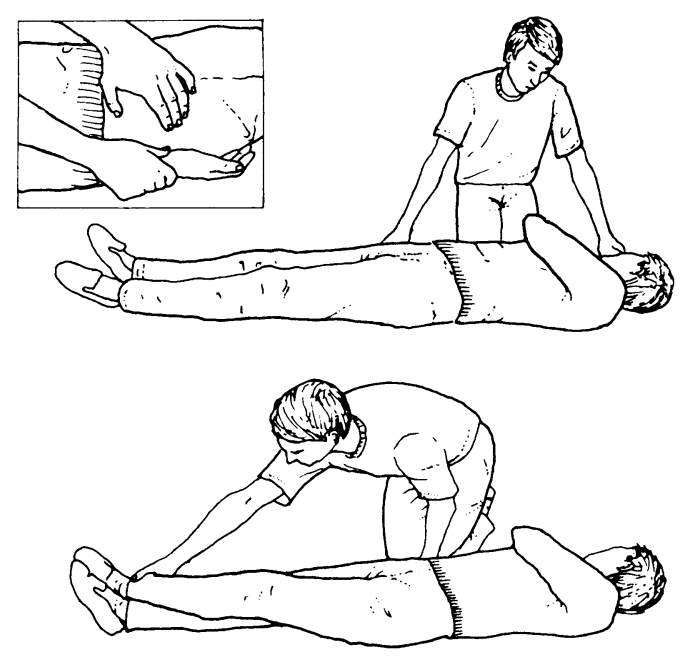

The patient whose airway is clear who is breathing spontaneously should be turned into the recovery position. This prevents the tongue falling back and reduces the risk of inhalation of gastric contents. There is no universally standard recovery position, but any position that secures the casualty on his or her side is acceptable provided the following requirements are met:

- The procedure should minimise the movement of the casualty

- The casualty's head, neck, and trunk should be kept in a straight line

- The position should permit gravity drainage of liquid from the casualty's mouth

- The position should be stable - that is, the casualty should not be able to fall over or topple into any other position. The position should be suitable for a casualty being carried on a stretcher.

The Resuscitation Council has adopted the position described in the fifth edition of the Combined First Aid Manual. ${ }^{5}$ 

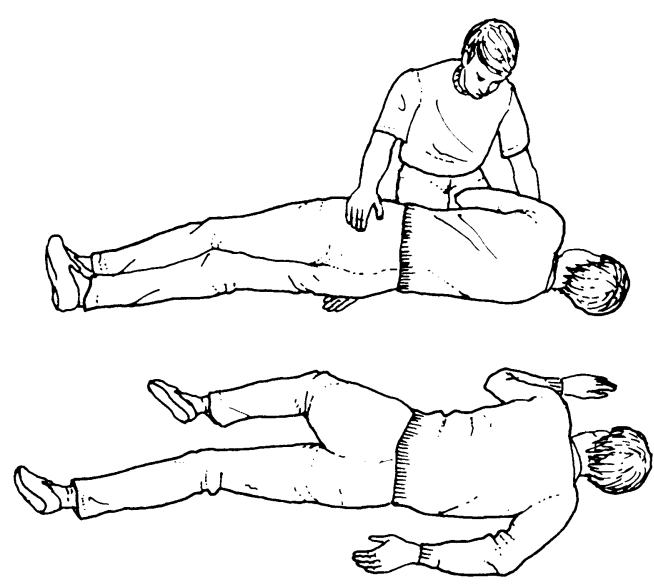

\section{Choking}

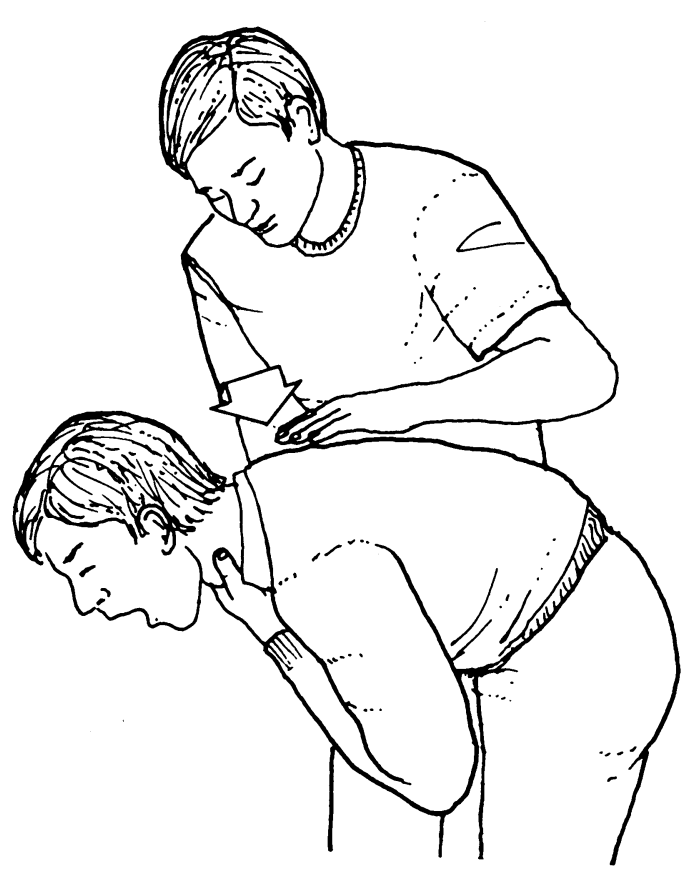

\section{Resuscitation by health care professionals}

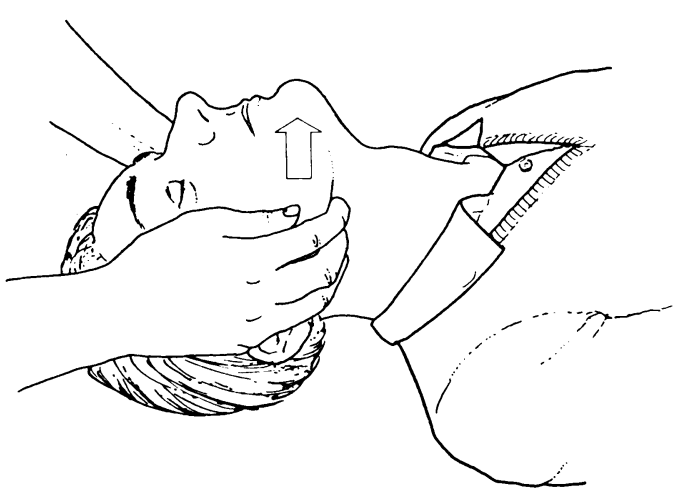

bsence of scientific evidence to the contrary, the recommendations of the simple, are as follows. A conscious, choking victim should be encouraged to cough. The victim should be bent forward-for example over a chair - to enlist the aid of gravity. If coughing fails to dislodge the obstruction the casualty should lean forward and blows should then be delivered to the middle of the upper back. The trained first aider
administer a series of abdominal thrusts (Heimlich manoeuvre). Alternating a series of back blows with a series of abdominal thrusts seems to be the most effective procedure.

An unconscious victim should be given abdominal or chest thrusts by direct compression.

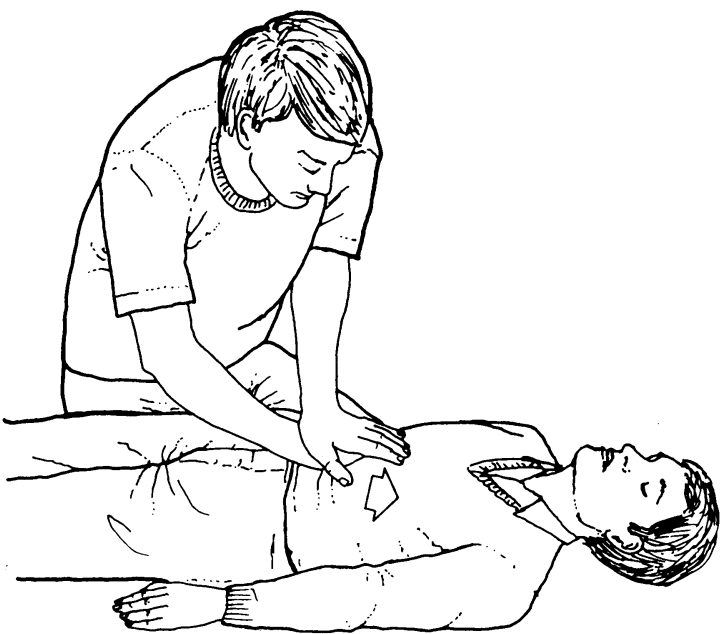

In witnessed collapse - for example in hospital - when a primary cardiac arrest can be presumed, professionals might wish to combine the initial assessment of airway, respirations, and pulse.

Controversy still surrounds the use of the precordial (chest) thump. There is evidence that ventricular fibrillation can be successfully terminated by a chest thump, ${ }^{7}$ but in a large study of out of hospital resuscitation attempts an appreciable proportion of patients were thumped to an untreatable rhythm. ${ }^{8}$ The chest thump seems to have a role in witnessed arrests, perhaps when a defibrillator is not immediately to hand or when the patient is already monitored. This is likely to limit its use to hospitals.

As well as the head tilt-chin lift technique the professional should be trained in the additional technique of jaw thrust. Jaw thrust alone should be used if an injury to the cervical spine is suspected.

Several "resuscitation devices", for example, face shields, and resuscitation airways have been produced for the use of emergency services staff and health care professionals with the aim of minimising any risk of 

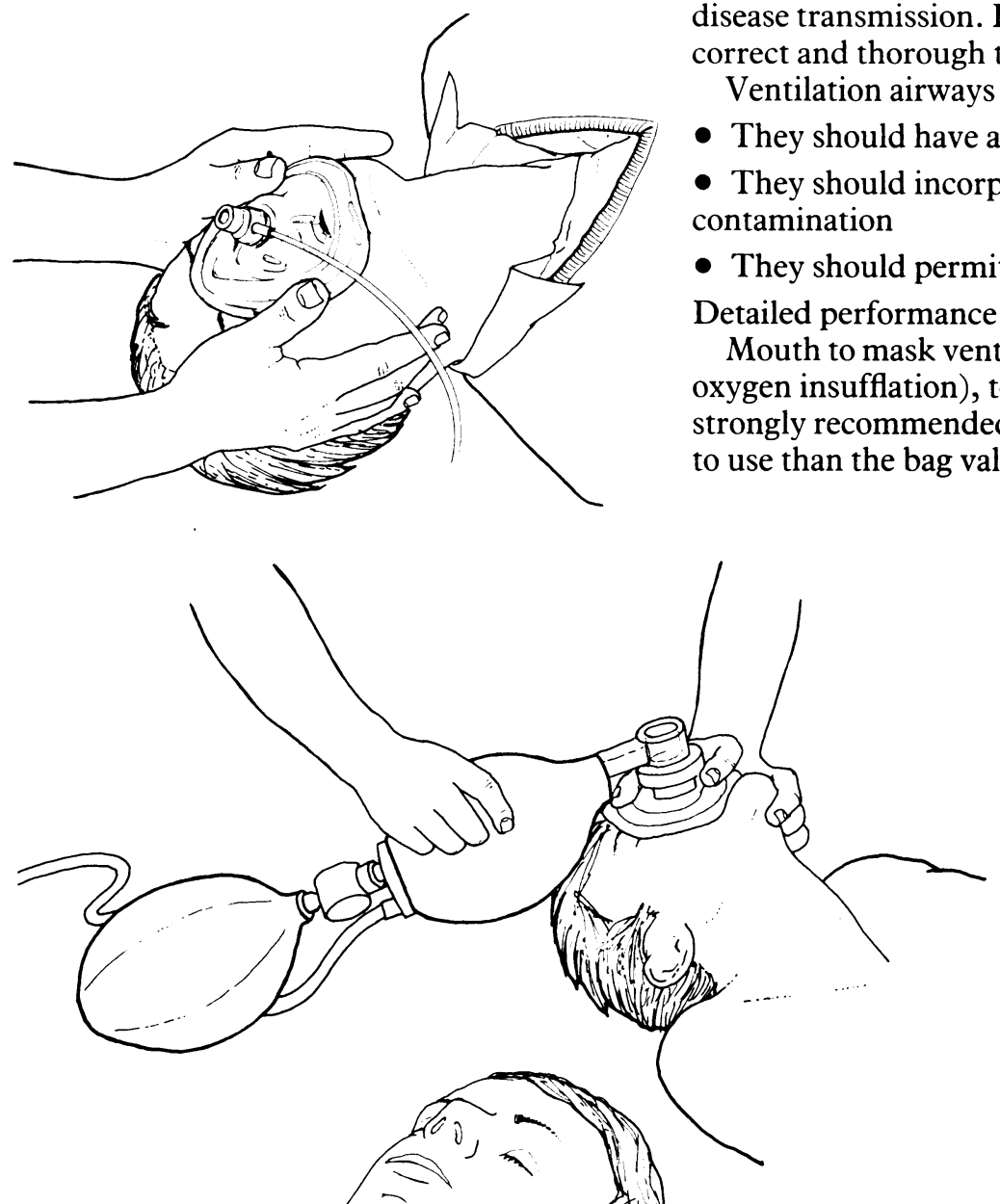
contamination disease transmission. People who carry such resuscitation aids must receive correct and thorough training in their use.

Ventilation airways should meet the following requirements:

- They should have a mimimum lumen diameter of $5 \mathrm{~mm}^{9}$

- They should incorporate a one way valve to minimise cross

- They should permit an adequate seal at the casualty's mouth.

Detailed performance criteria have not so far been specified.

Mouth to mask ventilation using a portable facemask (with supplemental oxygen insufflation), together with the use of an oropharyngeal airway, is strongly recommended for the professional rescuer. The technique is easier to use than the bag valve mask, and higher tidal volumes can be achieved. ${ }^{10}$

Bag valve mask units are incapable of delivering high concentrations of oxygen without the addition of an attached oxygen reservoir. This is particularly important for resuscitation in children.

Oxygen powered breathing devices must never be used by untrained people; the likelihood of complications is high and correct use requires extensive training and experience. Oxygen powered ventilators are not suitable for use in children. Commercial pressure cycled automatic ventilators should not be used in conjunction with chest compression because this may prematurely trigger the termination of the inflation cycle, resulting in inadequate ventilation. When oxygen powered ventilators are used with a facemask the potential for gastric distension is high; preferrably they should be used only in conjunction with a cuffed endotracheal tube.

Circulation - The ratio of compressions to ventilations for two rescuer cardiopulmonary resuscitation is 5:1 with a compression rate of 60-80 per minute. Whatever the rates and ratios chosen they must not compromise effective ventilation. Thus with two rescuer cardiopulmonary resuscitation there will normally be a pause between compression cycles for ventilation. When the airway is unprotected the use of the cricoid pressure manoeuvre to occlude the oesophagus and help prevent regurgitation is recommended. With the airway secured by endotracheal intubation the pause for ventilation between cycles becomes unnecessary. When the intubated patient is connected to an automatic ventilator both procedures (compression and ventilation) can take place independently and, perhaps, synchronously.

Basic life support is essentially a practical skill and students require practical training to learn it. At least two hours of training devoted mainly to manikin practice with one instructor to no more than six pupils is required to teach basic life support. Retention of skills is also poor unless regular retraining is carried out. ${ }^{11}{ }^{12}$ The Resuscitation Council strongly endorses the recommendations of the 1987 report of the Royal College of Physicians on the training and organisation required to provide effective resuscitation. ${ }^{13}$
1 Weaver WD, Cobb LA, Hallstrom AP, et al. Factors influencing survival after out-of-hospital cardiac arrest. $7 \mathrm{Am}$ Coll Cardiol 1986;7:752-7.

2 Guildner CW. Resuscitation - opening the airway. A comparative study of techniques for opening an airway obstructed by the tongue. Fournal of the American College of Emergency Physicians 1976;5:588-90.

3 Melker RJ. Recommendations for ventilation during cardiopulmonary resuscitation. Time for a change? Crit Care Med 1985;13:882-3.

4 American Heart Association. Standards and guidelines for cardiopulmonary resuscitation (CPR) and emergency cardiac care (ECC). FAMA 1986;255:2905-89.

5 St John Ambulance, St Andrew's Ambulance Association, The British Red Cross Society. First aid manual. London: Dorling Kindersley, 1987.

6 Montoya D. Management of the choking victim. Can Med Assoc f 1986;135:305-10

7 Caldwell G, Millar G, Quinn E, et al. Simple mechanical methods for cardioversion defence of the precordial thump and cough version. Br Med f 1985;291:627-30. 8 Miller JM, Tresch D, Horowitz L, et al. The precordial thump. Ann Emerg Med 1984;13:791-4.

9 Safar P, Penninckx J. Cricothyroid membrane puncture with special cannula Anesthesiology 1967;28:943-8.

10 Elling R, Politis J. An evaluation of emergency medical technicians' ability to use manual ventilation devices. Ann Emerg Med 1983;12:765-8.

11 Wynne G. Training and rentention of skills. In: Evans TR, ed. A B C of Resuscitation. London: British Medical Journal, 1986:32-5.

12 McKenna SP, Glendon AI. Occupational first aid training. Decay of CPR skills. Fournal of Occupational Psychology 1985;58:109-17.

13 Royal College of Physicians. Resuscitation from cardiopulmonary arrest. Training and organisation. $\mathcal{F} R$ Coll Physicians Lond 1987;21:1-8. 